\title{
MODULES SATISFYING BOTH CHAIN CONDITIONS WITH RESPECT TO A TORSION THEORY
}

\author{
JONATHAN S. GOLAN
}

\begin{abstract}
Goldman [3] has introduced the notion of the length of a module with respect to a torsion theory and has studied finitely-generated modules over left noetherian rings which have finite length. In this note we simplify the proofs of some of Goldman's results and generalize them by removing both the finite-generation and noetherianness conditions.
\end{abstract}

0 . Background and notation. Throughout the following, $R$ will denote an associative (but not necessarily commutative) ring with unit element 1 . We will denote by $R$-mod the category of all unitary left $R$-modules and will abuse notation by writing $M \in R$-mod when we mean to say that $M$ is an object of $R$-mod.

The set of all (hereditary) torsion theories on $R$-mod will be denoted by $R$-tors. The reader is referred to [2], [4], [7] for basic information about such theories. If $\tau \in R$-tors we denote the class of all $\tau$-torsion left $R$-modules by $\mathcal{T}_{\tau}$ and the class of all $\tau$-torsion-free left $R$-modules by $\mathcal{F}_{\tau}$. The $\tau$-torsion subfunctor of the identity functor on $R-\bmod$ is denoted by $T_{\tau}\left({ }_{-}\right)$. A submodule $N$ of a left $R$-module $M$ is said to be $r$-pure in $M$ if and only if $M / N$ is $r$-torsionfree. The set $R$-tors is a complete lattice when we define $\tau \leq \tau^{\prime}$ if and only if $\mathcal{T}_{\tau} \subseteq \mathcal{T}_{\tau^{\prime}}$ (or equivalently if and only if $\mathcal{F}_{\tau} \supseteq \mathcal{F}_{\tau^{\prime}}$ ) and when, for any subset $U$ of $R$-tors, $\wedge U$ is characterized by $\mathcal{T}_{\Lambda U}=\bigcap\left\{\mathcal{T}_{\tau} \mid \tau \in U\right\}$. For each $M \in R$ mod there is a unique largest element of $R$-tors relative to which $M$ is torsionfree. We denote this theory by $\chi(M)$.

Let $\tau \in R$-tors. A nonzero left $R$-module $M$ is said to be $\tau$-cocritical if and only if $M$ is $\tau$-torsion-free and, for any nonzero submodule $N$ of $M, M / N$ is $r$-torsion. Such modules are discussed, under various names, in [2] and [8]. Every nonzero submodule of a $\tau$-cocritical left $R$-module is $\tau$-cocritical and every $\tau$-cocritical left $R$-module is uniform. Moreover, a left $R$-module $M$ is $\tau$-cocritical for some torsion theory $\tau$ if and only if it is $\chi(M)$-cocritical.

It is not true that every $\tau \in R$-tors is of the form $\chi(M)$ for some $\tau$-cocritical left $R$-module $M$. If a torsion theory $\tau$ can be so represented then it is said to be prime [2]. Indeed, if $\tau$ is prime then $\tau=\chi(M)$ for every $\tau$-cocritical left $R$-module $M$. We call the set of all prime torsion theories on $R$-mod the left spectrum of $R$ and denote it by $R$-sp. If $\tau \in R$-tors we denote by $\operatorname{pgen}(\tau)$ the set of all prime torsion theories $\pi \in R$-sp satisfying $\pi \geq \tau$. (These are the prime generalizations of $\tau$.) It is not always true that, for a given torsion 
theory $\tau$, we have $\tau=\wedge$ pgen $(\tau)$. When this happens $\tau$ is said to be semiprime. If $\tau=\bigwedge U$ for some $U \subseteq\{\chi(M) \mid M \in R$-mod is $\tau$-cocritical $\}$ then $\tau$ is said to be strongly semiprime. The rings over which every torsion theory is semiprime and those over which every torsion theory is strongly semiprime are characterized in [6]. The latter are precisely the left seminoetherian rings, i.e. those rings having left Gabriel dimension.

Let $M$ be a left $R$-module. We define the support, $\operatorname{supp}(M)$, of $M$ to be $\operatorname{supp}(M)=\left\{\pi \in R\right.$-sp $\left.\mid M \notin \mathcal{T}_{\pi}\right\}$. See [1] for details. We define the assassin $\operatorname{ass}(M)$ of $M$ to be ass $(M)=\{\pi \in R$-sp/there exists a $\pi$-cocritical submodule of $M$ \}. See [1], [2], [8]. For any submodule $N$ of $M$, ass $(N) \subseteq \operatorname{ass}(M) \subseteq \operatorname{ass}(N)$ $\cup$ ass $(M / N)$. Moreover, if $M$ is $\pi$-cocritical for some $\pi \in R$-sp then $\operatorname{ass}(N)=$ $\{\pi\}$ for any nonzero submodule $N$ of $M$.

1. Modules which are both $\tau$-artinian and $\tau$-noetherian. Let $\tau \in R$-tors. Following Manocha [5], we say that a left $R$-module $M$ is $\tau$-artinian [resp. $\tau$ noetheriar] if and only if the set of all $r$-pure submodules of $M$ satisfies the descending chain condition [resp. the ascending chain condition]. The class of all $r$-artinian [resp. $r$-noetherian] left $R$-modules is a Serre class [5] and hence so is the class of all left $R$-modules which are both $r$-artinian and $r$ noetherian. We denote this class by $\mathbb{Q}_{\tau}$. Then $\mathcal{T}_{\tau} \subseteq \mathbb{Q}_{\tau}$ and for any left $R$ module $M, M \in \mathbb{P}_{\tau}$ if and only if $M / T_{\tau}(M) \in \mathbb{Q}_{\tau}$.

(1.1) Proposition. If $\tau, \tau^{\prime} \in R$-tors then:

(1) $\tau \leq \tau^{\prime} \Rightarrow \mathbb{Q}_{\tau} \subseteq \mathbb{Q}_{\tau^{\prime}}$.

(2) $\mathbb{Q}_{\tau \wedge \tau^{\prime}}=\mathbb{Q}_{\tau}^{\tau} \cap \mathbb{Q}_{\tau^{\prime}}^{\tau}$.

Proof. (1) Let $M \in \mathbb{Q}_{\tau}$. If $M_{1} \subseteq M_{2} \subseteq \ldots$ is an ascending chain of $\tau^{\prime}$ pure submodules of $M$ then $M / M_{i} \in \mathcal{F}_{\tau^{\prime}} \subseteq \mathcal{F}_{\tau}$ for each index $i$ and so there exists an index $k$ for which $M_{k}=M_{k+1}=\ldots$. Thus $M$ is $\tau^{\prime}$-noetherian. Similarly, $M$ is $\tau^{\prime}$-artinian and so $M \in \mathbb{Q}_{\tau^{\prime}}$.

(2) By (1), $\mathfrak{A}_{\tau \wedge \tau^{\prime}} \subseteq \mathfrak{A}_{\tau} \cap \mathfrak{A}_{\tau^{\prime}}$. Conversely, let $M \in \mathbb{A}_{\tau} \cap \mathfrak{A}_{\tau^{\prime}}$ and let $M_{1} \subseteq$ $M_{2} \subseteq \ldots$ be an ascending chain of $\left(\tau \wedge \tau^{\prime}\right)$-pure submodules of $M$. Then $M / M_{j}$ $\notin \mathcal{T}_{\tau} \cap \mathcal{T}_{\tau^{\prime}}$ for each index $j$ and so we can assume without loss of generality that there are an infinite number of indices $j$ for which $M / M_{j}$ is not $r$-torsion. By throwing away all of the other links in the chain we can in fact assume that $M / M_{j}$ is not $\tau$-torsion for all indices $j$.

For each index $j$, let $N_{j}$ be the submodule of $M$ defined by $N_{j} / M_{j}=$ $T_{\tau}\left(M / M_{j}\right)$. Then we have an ascending chain $N_{1} \subseteq N_{2} \subseteq \ldots$ of $\tau$-pure submodules of $M$ which must therefore terminate at some index $k$, i.e. $N_{k}=N_{k+1}=$ ... For each $j>k$, we have $M_{j} / M_{k} \subseteq N_{j} / M_{k}=N_{k} / M_{k} \in \mathcal{T}_{\tau}$. But $M_{j} / M_{k} \subseteq$ $M / M_{k} \in \mathcal{F}_{\tau \Lambda \tau^{\prime}}$ and so we must have $M_{j} / M_{k} \notin \mathcal{T}_{\tau^{\prime}}$ for all $j>k$. For each $j>k$ let $N_{j}^{\prime}$ be the submodule of $N_{k}$ defined by,$M_{j}=T_{\tau^{\prime}}\left(N_{k} / M_{j}\right)$. Then we have the ascending chain $N_{k}^{\prime} \subseteq N_{k+1}^{\prime} \subseteq \ldots$ of $\tau^{\prime}$-pure submodules of $N_{k}$. Since $M \in \mathbb{Q}_{\tau^{\prime}}$, we 
have $N_{k} \in \mathbb{Q}_{\tau^{\prime}}$ and so there exists an index $h$ for which $N_{h}^{\prime}=N_{h+1}^{\prime}=\ldots$. If $j>b$ then $M_{j} / M_{h} \subseteq N_{j}^{\prime} / M_{h}=N_{h}^{\prime} / M_{h} \in \mathcal{T}_{\tau^{\prime}}$ and so $M_{j} / M_{h} \in \mathcal{T}_{\tau}^{+1} \cap \mathcal{T}_{\tau^{\prime}}=$ $\mathcal{T}_{\tau \wedge \tau^{\prime}}$. But $M_{j} / M_{h} \subseteq M / M_{h} \in \mathcal{F}_{\tau \wedge \tau^{\prime}}$ and so we must have $M_{j}=M_{h}$. Therefore $M$ is $\left(\tau \wedge \tau^{\prime}\right)$-noetherian. A similar proof shows that $M$ is $\left(\tau \wedge \tau^{\prime}\right)$-artinian and so $M \in \mathbb{A}_{\tau \wedge \tau^{\prime}}$.

(1.2) Corollary. The following conditions are equivalent for $M \in R$-mod:

(1) $M \in \mathbb{Q}_{\boldsymbol{x}(M)}$.

(2) $M \in \mathbb{Q}_{\tau} \cap \mathcal{F}_{\tau}$ for some $\tau \in R$-tors.

Proof. (1) $\Rightarrow$ (2): Trivial. (2) $\Rightarrow(1)$ : If $M \in \mathbb{Q}_{\tau} \cap \mathcal{F}_{\tau}$ then $\chi(M) \geq \tau$ and so $M \in \mathbb{A}_{\boldsymbol{\chi}(M)}$ by Proposition 1.1(1).

Goldman [3] has shown that $M \in \mathbb{Q}_{\tau}$ if and only if there exists a chain $T_{\tau}(M)=M_{0} \subset M_{1} \subset \ldots \subset M_{n}=M$ of submodules of $M$ having the property that $M_{i+1} / M_{i}$ is $r$-cocritical for each $0 \leq i<n$. Such a chain is called a $r$-chain in $M$. Moreover, if $T_{\tau}(M)=M_{0}^{\prime} \subset M_{1}^{\prime} \subset \ldots \subset M_{k}^{\prime}=M$ is another $\tau$-chain in $M$ then $k=n$ and

$$
\left\{\chi\left(M_{i+1} / M_{i}\right) \mid 0 \leq i<n\right\}=\left\{\chi\left(M_{j+1}^{\prime} / M_{j}^{\prime}\right) \mid 0 \leq j<k\right\} .
$$

The integer $n$ is therefore unique and is called the $\tau$-length of $M$; it is denoted by $\operatorname{len}_{\tau}(M)$. The set $\left\{\chi\left(M_{i+1} / M_{i}\right) \mid 0 \leq i<n\right\}$ of prime torsion theories is called the set of $\tau$-invariants of $M$ and is denoted by $\operatorname{inv}_{\tau}(M)$. For each $M \in \mathbb{Q}_{\tau}$ we have

$$
\operatorname{len}_{\tau}(M)=\operatorname{len}_{\tau}\left(M / T_{\tau}(M)\right) \text { and } \operatorname{inv}_{\tau}(M)=\operatorname{inv}_{\tau}\left(M / T_{\tau}(M)\right) \text {. }
$$

Moreover, Goldman [3, Corollary 2.8] showed that if $M \in \mathbb{Q}_{\tau}$ then $\operatorname{inv}_{\tau}(M)=$ $\operatorname{pgen}(\tau) \cap \operatorname{supp}(M)$. Thus, if $\tau \leq \tau^{\prime}$ we have $\operatorname{inv}_{\tau}(M) \supseteq \operatorname{inv}_{\tau},(M)$.

For each $M \in R$-mod and each $\tau \in R$-tors let $W(M, \tau)$ be the set of all prime torsion theories $\pi$ satisfying both of the following conditions:

(1) $\pi$ is a minimal element of $\operatorname{pgen}(\tau)$;

(2) $\pi$ is a maximal element of $\operatorname{supp}(M)$.

(1.3) Proposition. If $\tau \in R$-tors and $M \in \mathbb{Q}_{\tau}$ then $\operatorname{inv}_{\tau}(M)=W(M, \tau)$.

Proof. We have already noted that $\operatorname{inv}_{\tau}(M)=\operatorname{pgen}(\tau) \cap \operatorname{supp}(M) \supseteq W(M, \tau)$. Conversely, if $\pi \in \operatorname{inv}_{\tau}(M)$ and if $\tau^{\prime} \in R$-tors satisfies $\tau^{\prime}>\pi$ we want to show that $\tau^{\prime} \notin \operatorname{supp}(M)$. Assume the contrary. Then $0 \neq N=M / T_{\tau^{\prime}}(M) \epsilon$ $\mathcal{F}_{\tau^{\prime}} \subseteq \mathcal{F}_{\pi} \subseteq \mathfrak{F}_{\tau}$. Moreover, $M \in \mathbb{Q}_{\tau}$ implies that $N \in \mathbb{A}_{\tau}$. If $0=N_{0} \subset \ldots \subset$ $N_{k}=N$ is a $\tau$-chain in $N$ then $N_{1}$ is a $\tau$-cocritical submodule of $N$ and so $\chi\left(N_{1}\right)$ is a minimal element of $\operatorname{pgen}(\tau)$ for if $\pi^{\prime \prime} \in \operatorname{pgen}(\tau)$ and $\pi^{\prime \prime} \leq \chi\left(N_{1}\right)$, then $N_{1} \in \mathcal{F}_{\boldsymbol{\chi}\left(N_{1}\right)} \subseteq \mathcal{F}_{\pi^{\prime \prime}}$. On the other hand, for any nonzero submodule $N^{\prime}$ of $N_{1}, N_{1} / N^{\prime} \in \mathcal{T}_{\tau} \subseteq \mathcal{T}_{\pi^{\prime \prime}}$. Therefore $N_{1}$ is also $\pi^{\prime \prime}$-cocritical and so $\pi^{\prime \prime}=$ $\chi\left(N_{1}\right)$. But $\chi\left(N_{1}\right) \geq \chi(N) \geq \tau^{\prime}>\pi \in \operatorname{pgen}(\tau)$, a contradiction. Therefore we must have $\tau^{\prime} \notin \operatorname{supp}(M)$ and so $\pi$ is a maximal element of $\operatorname{supp}(M)$. 
We now want to show that $\pi$ is a minimal element of pgen $(\tau)$. If $T_{\tau}(M)=$ $M_{0} \subset M_{1} \subset \ldots \subset M_{n}=M$ is a $r$-chain in $M$ then there exists an index $k, 0 \leq k$ $<n$, for which $\pi=\chi\left(M_{k+1} / M_{k}\right)$, where $M_{k+1} / M_{k}$ is a $r$-cocritical left $R$-module. But we have already seen that this suffices to prove that $\pi$ is minimal in $\operatorname{pgen}(\tau)$. Therefore $\pi \in W(M, \tau)$ and so we have that $\operatorname{inv}_{\tau}(M)=W(M, \tau)$.

(1.4) Corollary. If $M \in \mathbb{Q}_{\tau}$ for some torsion theory $\tau$ then $M \in \mathcal{T}_{\tau}$ if and only if $W(M, \tau)=\varnothing$.

(1.5) Proposition. Let $M \in R$-mod and let $\tau \in R$-tors be semiprime. Then the following conditions are equivalent:

(1) $M \in \mathbb{Q}_{\tau}$.

(2) $W(M, \tau)$ is a finite set and $M \in \mathbb{Q}_{\pi}$ for each $\pi \in W(M, \tau)$.

Proof. $(1) \Rightarrow(2)$ : By Proposition 1.3, (1) implies that $W(M, \tau)=\operatorname{inv}_{\tau}(M)$ and the latter is a finite set. By Proposition $1.1(1), M \in \mathbb{A}_{\pi}$ for each $\pi \geq \tau$ and so in particular for each $\pi \in W(M, \tau)$.

$(2) \Rightarrow(1)$ : Let $U=\operatorname{pgen}(\tau) \backslash W(M, \tau)$. Then $M \in \mathcal{J}_{\wedge U} \subseteq \mathbb{Q}_{\Lambda U}$. Moreover, since $\tau$ is semiprime we have $\tau=\wedge$ pgen $(\tau)=[\wedge U] \wedge[\wedge W(M, \tau)]$. Since $W(M, \tau)$ is a finite set and since $M \in \mathbb{Q}_{\pi}$ for each $\pi \in W(M, \tau)$, we have $M \epsilon$ $\mathbb{Q}_{\tau}$ by Proposition 1.1(2).

2. Modules having finite intrinsic length. In Corollary 1.2 we saw the importance of asking when a left $R$-module $M$ belongs to $\mathbb{A}_{\boldsymbol{\chi}(M)}$. Goldman [3] calls modules having this property modules with finite intrinsic length.

(2.1) Proposition. If $0 \neq M \in \mathbb{A}_{\mathbf{x}(M)}$ then:

(1) $\varnothing \neq \operatorname{ass}(M)=\operatorname{inv}_{\boldsymbol{x}(M)}(M)$;

(2) $\operatorname{ass}(M)$ is a finite set;

(3) there exists a large submodule of $M$ of the form $\bigoplus_{i=1}^{n} N_{i}$ where the $N_{i}$ are cyclic cocritical submodules of $M$;

(4) $M$ has finite uniform dimension;

(5) to each $\pi \in \operatorname{ass}(M)$ we can associate a submodule $N_{\pi}$ of $M$ such that the following conditions are satisfied:

(i) $\operatorname{ass}\left(M / N_{\pi}\right)=\{\pi\}$,

(ii) $\operatorname{ass}\left(N_{\pi}\right)=\operatorname{ass}(M) \backslash\{\pi\}$;

(iii) $0=\bigcap\left\{N_{\pi} \mid \pi \in\right.$ ass $\left.(M)\right\}$ and this intersection is reduced;

(6) $\chi(M)=\bigwedge \operatorname{ass}(M)$;

(7) $\chi(M)$ is strongly semiprime.

Proof. We will first prove that for any left $R$-module $M^{\prime} \in \mathcal{F}_{\boldsymbol{\chi}(M)} \cap \mathcal{C}_{\boldsymbol{\chi}(M)}$ we have ass $\left(M^{\prime}\right) \subseteq \operatorname{inv}_{\boldsymbol{\chi}(M)}\left(M^{\prime}\right)$. This will prove one inclusion of (1). The proof of the reverse inclusion will be delayed until later. We will proceed by induction on $\operatorname{len}_{\chi(M)}\left(M^{\prime}\right)$. If $\operatorname{len}_{\chi(M)}\left(M^{\prime}\right)=1$ then $M^{\prime}$ is $\chi(M)$-cocritical

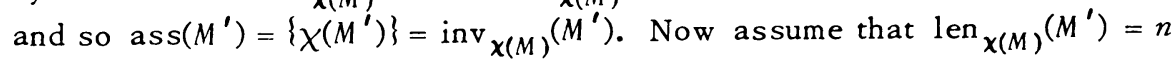


and that the result has been established for all $\chi(M)$-torsion-free modules the $\chi(M)$-length of which is less than $n$. Let $0=M_{0}^{\prime} \subset M_{1}^{\prime} \subset \ldots \subset M_{n}^{\prime}=M^{\prime}$ be a $\chi(M)$-chain in $M^{\prime}$. Then $M^{\prime} / M_{1}^{\prime}$ is $\chi(M)$-torsion-free [3] and $\operatorname{len}_{\chi(M)}\left(M^{\prime} / M_{1}^{\prime}\right)=n-1$. Moreover, $\operatorname{inv}_{\boldsymbol{\chi}(M)}\left(M^{\prime}\right)=\left\{\chi\left(M_{1}\right)\right\} \cup \operatorname{inv}_{\boldsymbol{\chi}(M)}\left(M^{\prime} / M_{1}^{\prime}\right)$. Let $\pi \in \operatorname{ass}\left(M^{\prime}\right)$. There then exists a submodule $N$ of $M^{\prime}$ for which $\pi=$ $\chi(N)$. If $N \cap M_{1}^{\prime} \neq 0$ then $N \cap M_{1}^{\prime}$ is $\pi$-cocritical and so $\pi=\chi\left(N \cap M_{1}^{\prime}\right)=$ $\chi\left(M_{1}^{\prime}\right) \in \operatorname{inv}_{\chi(M)}\left(M^{\prime}\right)$. If $N \cap M_{1}^{\prime}=0$ then $N \cong\left[N+M_{1}^{\prime}\right] / M_{1}^{\prime} \subseteq M^{\prime} / M_{1}^{\prime}$ and so $\pi \epsilon$ ass $\left(M^{\prime} / M_{1}^{\prime}\right)$. By induction we then have $\pi \in \operatorname{inv}_{\chi(M)}\left(M^{\prime} / M_{1}^{\prime}\right) \subseteq \operatorname{inv}_{\chi(M)}\left(M^{\prime}\right)$.

In particular, we have ass $(M) \subseteq \operatorname{inv}_{x(M)}(M)$ which is one direction of (1). Since $\operatorname{inv}_{\boldsymbol{\chi}(M)}(M)$ is a finite set, we immediately have (2).

The module $M$ certainly has at least one cocritical submodule $N$ (i.e. $N$ is $\tau$-cocritical for some $\tau \in R$-tors), namely the first nonzero link in a $\chi(M)$-chain in $M$. Therefore $\varnothing \neq \operatorname{ass}(M)$. If $0 \neq x \in N$ then $R x$ is also cocritical and so without loss of generality we can assume that $N$ is a cyclic left $R$-module. Consider the set of all submodules of $M$ of the form $\bigoplus_{i \in \Lambda} N_{i}$ where the $N_{i}$ are cyclic cocritical submodules of $M$. This set is clearly inductive and so by Zorn's lemma it contains a maximal element $M^{\prime}=\bigoplus_{i \in \boldsymbol{\Omega}} N_{i}$. We claim that $M^{\prime}$ is a large submodule of $M$. Indeed, if not then there exists a nonzero submodule $M^{\prime \prime}$ of $M$ for which $M^{\prime \prime} \cap M^{\prime}=0$. By Proposition 1.1, $M^{\prime \prime} \in \mathbb{Q}_{\chi(M)}$ and so $M^{\prime \prime}$ has a nonzero cocritical submodule $N^{\prime \prime}$, which we can again assume to be cyclic. Therefore $M^{\prime} \oplus N^{\prime \prime}$. is strictly larger than $M^{\prime}$, contradicting the maximality of $M^{\prime}$. Thus $M^{\prime}$ is large in $M$. We now want to show that the set $\Omega$ is finite. Assume not. Then without loss of generality we can assume that $\Omega$ contains the set of natural numbers. For each natural number $i$, let $Y_{i}=\bigoplus_{j=1}^{i} N_{j}$. Then for each index $i, M^{\prime} / Y_{i}$ is isomorphic to a submodule of $M^{\prime}$ and so is $\chi(M)$-torsion-free. Hence we have an infinite ascending chain $Y_{1} \subset Y_{2} \subset \ldots$ of $\chi(M)$-pure submodules of $M^{\prime}$. But if $M \in \mathbb{A}_{\chi(M)}$ then $M^{\prime} \in \mathbb{A}_{\chi(M)}$, a contradiction. Therefore $\Omega$ is a finite set and so we have proven (3). As an immediate consequence of this we also have (4).

Now let $\pi \in \operatorname{ass}(M)$ and consider the class of all submodules $N^{\prime}$ of $M$ for which $\pi \notin \operatorname{ass}\left(N^{\prime}\right)$. This set is clearly nonempty and by [8, Proposition 3.1] it is inductive. Therefore by Zorn's lemma there is a maximal such module $N_{\pi}$ which clearly satisfies (5)(ii).

Let $\pi^{\prime} \epsilon \operatorname{ass}\left(M / N_{\pi}\right)$. Then there exists a $\pi^{\prime}$-cocritical submodule $M^{\prime} / N_{\pi}$ of $M / N_{\pi}$. Thus

$$
\operatorname{ass}\left(M^{\prime}\right) \subseteq \operatorname{ass}\left(N_{\pi}\right) \cup \operatorname{ass}\left(M^{\prime} / N_{\pi}\right)=\operatorname{ass}\left(N_{\pi}\right) \cup\left\{\pi^{\prime}\right\}
$$

and so, by the maximality of $N_{\pi}$, we have $\pi^{\prime}=\pi$. This establishes (5)(i).

Now let $N^{\prime}=\bigcap\left\{N_{\pi} \mid \pi \in \operatorname{ass}(M)\right\}$. Then $\operatorname{ass}\left(N^{\prime}\right) \subseteq \operatorname{ass}\left(N_{\pi}\right)$ for all $\pi \epsilon$ $\operatorname{ass}(M)$ and so ass $\left(N^{\prime}\right)=\varnothing$. But $M \in \mathbb{Q}_{\chi(M)}$ implies that $N^{\prime} \in \mathbb{Q}_{\boldsymbol{\chi}(M)}$ and 
so by what we proved above this implies that $N^{\prime}=0$. If $U$ is a proper subset of $\operatorname{ass}(M)$ and if $N^{\prime \prime}=\bigcap\left\{N_{\pi} \mid \pi \in U\right\}$, then $\operatorname{ass}\left(N^{\prime \prime}\right)=\operatorname{ass}(M) \backslash U \neq \varnothing$ and so $N^{\prime \prime} \neq 0$. This proves $(5)($ iii) and so completes the proof of (5).

If $\pi \in \operatorname{ass}(M)$ then $\pi \in \operatorname{inv}_{\boldsymbol{x}(M)}(M) \subseteq \operatorname{pgen}(\chi(M))$. Therefore $\bigwedge$ ass $(M) \geq$ $\chi(M)$. On the other hand, by (5) there exists a canonical monomorphism $M \rightarrow$ $M^{\prime}=\bigoplus\left\{M / N_{\pi} \mid \pi \in \operatorname{ass}(M)\right\}$. Then $\chi(M) \geq \chi\left(M^{\prime}\right)=\Lambda$ ass $(M)$ by [2, Proposition 5.4] and so $\chi(M)=\bigwedge$ ass $(M)$, proving (6).

In particular, (6) implies that $\chi(M)$ is semiprime. Since ass $(M) \subseteq$ $\operatorname{inv}_{\chi(M)}(M)$, each $\pi \in \operatorname{ass}(M)$ is of the form $\chi(N)$ where $N$ is a $\chi(M)$-cocritical left $R$-module. This implies that $\chi(M)$ is strongly semiprime, proving (7).

Finally, we return to prove the other direction of (1). Let $\pi \in \operatorname{inv}_{\boldsymbol{x}(M)}(M)$. Then $\pi \geq \chi(M)=\operatorname{\Lambda ass}(M)$. By (2), ass $(M)$ is a finite set of prime torsion theories and so by [3, Lemma 3.5] there exists a $\pi^{\prime} \epsilon$ ass $(M)$ for which $\pi \geq$ $\pi^{\prime}$. But by Proposition $1.3, \pi$ and $\pi^{\prime}$ are both maximal elements of $\operatorname{supp}(M)$ and so we must have $\pi=\pi^{\prime}$. Therefore $\pi \in \operatorname{ass}(M)$ and so $\operatorname{inv}_{\boldsymbol{x}(M)} \subseteq \operatorname{ass}(M)$, proving equality.

(2.2) Corollary. The following conditions are equivalent for $0 \neq M \in R$ mod:

(1) $M \in \mathbb{Q}_{\chi(M)}$.

(2) (i) $\chi(M)$ is strongly semiprime;

(ii) $W(M, \chi(M))$ is a finite set;

(iii) $M \in \mathbb{Q}_{\pi}$ for each $\pi \in W(M, \chi(M))$.

Proof. By Propositions 2.1 and 1.5.

\section{REFERENCES}

1. J. Golan, Topologies on the torsion-theoretic spectrum of a noncommutative ring, Pacific J. Math. 51 (1974), 439-450.

2. O. Goldman, Rings and modules of quotients, J. Algebra 13 (1969), 10-47. MR $39 \# 6914$.

3. - Elements of non-commutative arithmetic. I, 1974 (preprint).

4. J. Lambek, Torsion theories, additive semantics, and rings of quotients, Lecture Notes in Math., vol. 177, Springer-Verlag, Berlin and New York, 1971. MR 44 \#1685.

5. J. N. Manocha, Finiteness conditions and torsion theories. I, 1974 (preprint).

6. J. Raynaud, Localisations et anneaux semi-noethériens à droite, Publ. Dépt. Math. Lyon 8-2, 77-112.

7. B. Stenström, Rings and modules of quotients, Lecture Notes in Math., vol. 237, Springer-Verlag, Berlin and New York, 1971. MR 48 \#4010.

8. H. Storrer, On Goldman's primary decomposition, Lectures on Rings and Modules, Lecture Notes in Math., vol. 246, Springer-Verlag, Berlin and New York, 1972.

DEPARTMENT OF MATHEMATICS, UNIVERSITY OF HAIFA, HAIFA, ISRAEL 